\title{
Systemic mastocytosis as a risk factor for severe Hymenoptera sting- induced anaphylaxis
}

Ivan Alvarez-Twose, Patrizia Bonadonna, Almudena Matito, Roberta Zanotti, David Gonzalezde-Olano, Laura Sanchez-Muñoz, Jose Mario Morgado, Alberto Orfao, Luis Escribano

To the Editor:

We read with interest the recently published article by Stoevesandt et al about the over- and underestimated parameters in Hymenoptera-induced anaphylaxis. In this article, the authors report that the absence of urticaria/angioedema is an indicator for severe anaphylaxis and possibly also for mastocytosis, requiring assessment of baseline serum tryptase. Despite the large number of cases analyzed, authors mainly focused their goal on the evaluation of risk factors for severe anaphylaxis due to Hymenoptera field stings, particularly as regards details related to the sting reactions and concurrent medication. Conversely, they did not screen for mastocytosis in their series, and the potential association between the absence of hives during anaphylaxis and mastocytosis was supported only by studies including a limited number of cases, most of which had urticaria pigmentosa (UP). Because of this, we call the authors' and readers' attention to several previous studies that have investigated the association between anaphylaxis and mastocytosis. On the basis of a multivariate logistic regression analysis, we have previously identified risk factors associated with systemic mastocytosis (SM) in patients with severe mast cell (MC) mediator-release symptoms including cases with anaphylaxis referred to the Reference Center of the Spanish Network on Mastocytosis (REMA). In our study published in 2010, a simple and efficient score model for predicting SM was proposed (REMA score), which was further validated prospectively in the largest series of patients with SM lacking UP reported so far. In these studies, we already demonstrated that the absence of urticaria and angioedema and the presence of dizziness and/or syncope during anaphylaxis, together with increased baseline serum tryptase levels and male gender, are independent predictors for an underlying SM in these patients. Noteworthy, all patients included in our studies lacked UP, which further emphasizes the clinical utility of the REMA score and demonstrated that the severity of anaphylaxis is most likely due to the presence of clonal MC rather than the absence of cutaneomucosal symptoms itself. Similarly, elevated baseline serum tryptase levels in patients with a history of anaphylaxis should be rather considered as an epiphenomenon of the enhanced MC activation and/or relatively increased MC numbers than a true risk factor for the severity of anaphylaxis, such risk being most frequently conferred by an underlying clonal MC disorder. Table I details the distribution of the REMA score parameters in our updated series of patients with hymenoptera-induced anaphylaxis $(n=71)$ who were screened for SM. It should be emphasized that SM without UP associated to anaphylaxis is characterized by low bone marrow (BM) MC burden, which typically results in the absence of BM MC aggregates in a significant proportion of patients. In this scenario, cytomorphological characterization of $\mathrm{MC}$ in BM smears is frequently hampered by their limited numbers and their preferential location within BM particles; therefore, highly-sensitive diagnostic methods including multiparameter flow cytometry immunophenotyping for the identification of low numbers of aberrant BM MCs, together with analysis of the KIT mutation in highly purified BM MC fractions, 7 become specially relevant in the study of suspected clonal MC disorders without UP associated to anaphylaxis. 
TABLE I. Distribution of parameters included in the REMA score in 71 patients with Hymenoptera sting anaphylaxis in the absence of UP, according to the presence versus absence of an underlying SM

$\begin{array}{lccc} & \text { ISM patients } & \text { Non-ISM patients } & \\ \text { Male gender } & (\mathrm{n}=60) & (\mathrm{n}=11) & \text { P value } \\ \text { Urticaria* }^{*} & 51(85) & 7(63) & \mathrm{NS} \\ \text { Angioedema* }^{*} & 12(20) & 6(55) & .02 \\ \text { Dizziness* }^{*} & 3(5) & 7(64) & <.001 \\ \text { Syncope* }^{*} & 51(85) & 8(73) & \mathrm{NS} \\ \text { BST } & 41(68) & 1(9) & <.001 \\ \text { Median score value (range) } & 22.4(6.8-217) & 15(4.6-39.5) & .006 \\ \text { (2) } & 5(0-7) & 1(-4 \text { to } 7) & .001\end{array}$

Note. Results expressed as number of cases and percentage in parentheses or as median and range between parentheses.

BST, Baseline serum tryptase; ISM, indolent SM without skin lesions; NS, not significant.

*Clinical symptoms observed during acute anaphylactic episodes. 